\title{
Best Equivariant Estimator of Extreme Quantiles in the Multivariate Lomax Distribution
}

\author{
N. Sanjari Farsipour \\ Department of Statistics, College of Mathematical Sciences, Alzahra University, Tehran, Iran \\ Email: sanjari n@yahoo.com
}

Received 6 November 2013; accepted 27 June 2015; published 30 June 2015

Copyright (C) 2015 by author and Scientific Research Publishing Inc.

This work is licensed under the Creative Commons Attribution International License (CC BY). http://creativecommons.org/licenses/by/4.0/

(c) (i) Open Access

\section{Abstract \\ The minimum risk equivariant estimator of a quantile of the common marginal distribution in a multivariate Lomax distribution with unknown location and scale parameters under Linex loss function is considered.}

\section{Keywords}

Best Affine Equivariant Estimator, Quantile Estimation, Lomax (Pareto II) Distributions, Linex Loss Function

\section{Introduction}

In the analysis of income data, lifetime contexts, and business failure data the univariate Lomax (Pareto II) distribution with density $\frac{r}{\sigma}\left(1+\frac{x-\mu}{\sigma}\right)^{-1-r} ; x>\mu$, is a useful model [1]. The lifetime of a decreasing failure rate component may be describe by this distribution. It has been recommended by [2] as a heavy tailed alternative to the exponential distribution. The interested reader can see [3] and [4] for more details.

A multivariate generalization of the Lomax distribution has been proposed by [5] and studied by [6]. It may be obtained as a gamma mixture of independent exponential random variables in the following way. Consider a system of n components. It is then reasonable to suppose that the common operating environment shared by all components induces some kind of correlation among them. If for a given environment $\tau$, the component lifetimes $X_{1}, X_{2}, \cdots, X_{n}$ are independently exponentially distributed $E(\mu, \sigma / \tau)$ with density $\frac{\tau}{\sigma} \exp \left\{-\frac{\tau}{\sigma}(x-\mu)\right\}, x>\mu$, and the changing nature of the environment is accounted by a distribution function 
$\mathrm{F}($.$) , then the unconditional joint density of X_{1}, X_{2}, \cdots, X_{n}$ is

$$
f_{0}\left(x_{1}, x_{2}, \cdots, x_{n} ; \mu, \sigma\right)=\int_{0}^{\infty} \frac{\tau^{n}}{\sigma^{n}} \exp \left\{-\frac{\tau}{\sigma} \sum_{i=1}^{n}\left(x_{i}-\mu\right)\right\} I_{(\mu, \infty)}\left(x_{(1)}\right) \mathrm{d} F(\tau)
$$

where $x_{(1)}=\min \left\{x_{1}, x_{2}, \cdots, x_{n}\right\}$. Furthermore, if $F(\cdot)$ is a gamma distribution $G(r, 1)$ with density $\frac{1}{\Gamma(r)} \tau^{r-1} \mathrm{e}^{-\tau} ; r>0$, then (1) become

$$
f_{1}\left(x_{1}, x_{2}, \cdots, x_{n} ; \mu, \sigma\right)=\frac{\Gamma(n+r)}{\Gamma(r) \sigma^{n}} \frac{1}{\left(1+\frac{1}{\sigma} \sum_{i=1}^{n}\left(x_{i}-\mu\right)\right)} I_{(\mu, \infty)}\left(x_{(1)}\right) .
$$

This is called multivariate Lomax $M_{n}(r, \mu, \sigma)$ with location parameter $\mu$ and scale parameter $\sigma$. The same distribution is referred to as Mardia's multivariate Pareto II distribution, see [3] and [7]. If take $\mu=0$ and assign a different scale parameter, $\sigma_{i}$ to each $X_{i}$ we have

$$
f_{2}\left(x_{1}, x_{2}, \cdots, x_{n} ; \sigma_{i}\right)=\frac{\Gamma(n+r)}{\Gamma(r) \prod_{i=1}^{n} \sigma_{i}} \cdot \frac{1}{\left(1+\sum_{i=1}^{n} \frac{x_{i}}{\sigma_{i}}\right)^{n+r}} I_{(\mu, \infty)}\left(x_{(1)}\right) .
$$

For more information about the work on this distribution, the reader can see [8].

\section{Best Affine Equivarient Estimator}

Let $X_{1}, X_{2}, \cdots, X_{n} ; n \geq 2$ are from a multivariate Lomax distribution $M L_{n}(r, \mu, \sigma)$ with unknown $\mu$ and $\sigma$ and known $r$. We consider the linear function $\theta=\mu+k \sigma$ for given $k \geq 0$. When $k=p^{-1 / r}-1 ; 0<p<1$, $\theta$ is the $100(1-p)$ th quantile of the marginal distribution of $X_{i}$. Quantile estimation is of interest in reliability theory and lifetesting. [9] generalized results in [10] to a strictly Convex loss.

In this paper we consider the Linex loss function

$$
L(\theta, \delta)=\mathrm{e}^{a\left(\frac{\delta-\theta}{\sigma}\right)}-a\left(\frac{\delta-\theta}{\sigma}\right)-1
$$

where $a \neq 0$ is the shape parameter, which was introduced by [11] and was extensively used by [12].

The minimal sufficient statistic in the model (2) is $(S, X)$ where, $S=\sum_{i=1}^{n}\left(X_{i}-X_{(1)}\right)$ and $X=X_{(1)}$. Conditional on $\tau$, a random variable with $G(r, 1)$ distribution, $S$ and $X$ are independent with

$$
S\left|\tau \sim G\left(n-1, \frac{\sigma}{\tau}\right), \quad X\right| \tau \sim E\left(\mu, \frac{\sigma}{n \tau}\right) .
$$

So, the density of $(S, X)$ is

$$
\begin{aligned}
f(s, x ; \mu, \sigma) & =\int_{0}^{\infty} \frac{1}{(n-2) !} \frac{\tau^{n-1}}{\sigma^{n-1}} s^{n-2} \mathrm{e}^{-\frac{\tau s}{\sigma}} \frac{n \tau}{\sigma} \mathrm{e}^{\frac{n \tau}{\sigma}(x-\mu)} \frac{1}{\Gamma(r)} \tau^{r-1} \mathrm{e}^{-\tau} \mathrm{d} t \\
& =\frac{n \Gamma(n+r)}{(n-2) ! \Gamma(r) \sigma^{n}} \cdot \frac{s^{n-2}}{\left[1+\frac{1}{\sigma}\{s+n(x-\mu)\}\right]^{n+r}} ; \quad x>\mu, s>0
\end{aligned}
$$

The problem of estimating $\theta=\mu+k \sigma ; k \geq 0$ under the loss (4) is invariant under the affine group of transformations $(S, X) \rightarrow(c S, c X+b)$ and the equivariant estimator have the form $\delta=X+c S$ where c is a real constant.

Following [13], we study scale equivariant estimators of the form $\delta=\phi(Z) S$, where $Z=\frac{X}{S}$ and $\phi($.$) is$ 
a measurable function. Thus the equivariant estimator is of the form $\phi(Z) S$, where $\phi(Z)=Z+c$. Now, consider the risk of the estimator $X+c S$ for estimating $\mu+k \sigma$ when the loss is (4).

$$
\begin{aligned}
R(\theta, \delta) & =E\left\{\mathrm{e}^{a\left(\frac{X+c S-\mu-K \sigma}{\sigma}\right)}-a\left(\frac{X+c S-\mu-K \sigma}{\sigma}\right)-1\right\} \\
& =\mathrm{e}^{-a \frac{\mu+K \sigma}{\sigma}} E\left[\mathrm{e}^{\left.a\left(\frac{X+c S}{\sigma}\right)\right]-\frac{a}{\sigma} E(X)-\frac{a c}{\sigma} E(S)+\frac{a \mu}{\sigma}+a k-1}\right. \\
& =\mathrm{e}^{-\frac{a \mu}{\sigma}-a k} E\left\{E\left[\mathrm{e}^{\frac{a}{\sigma}(X+c S)} \mid \tau\right]\right\}-\frac{a}{\sigma} E\{E(X \mid \tau)\}-\frac{a c}{\sigma} E[E(S \mid \tau)]+\frac{a \mu}{\sigma}+a k-1 .
\end{aligned}
$$

Now, since $\tau \sim G(r, 1)$ and $X \mid \tau \sim E\left(\mu, \frac{\sigma}{n \tau}\right)$ and $S \mid \tau \sim G\left(n-1, \frac{\sigma}{\tau}\right)$ we have

$$
R(\theta, \delta)=n \mathrm{e}^{-a k} E_{\tau}\left\{\frac{\tau^{n}}{(n \tau-a)(\tau-a c)^{n-1}}\right\}-\frac{a}{\sigma}\left\{\frac{n r \mu}{\sigma}+1\right\}-\frac{\operatorname{acr}(n-1)}{\sigma}+\frac{a \mu}{\sigma}+a k-1
$$

which is finite if $r>a c$. By the invariant property of the problem we can take $(\mu, \sigma)=(0,1)$ and the risk becomes

$$
R((0,1), \delta)=n \mathrm{e}^{-a k} E_{\tau}\left\{\frac{\tau^{n}}{(n \tau-a)(\tau-a c)^{n-1}}\right\}-a-\operatorname{acr}(n-1)+a k-1
$$

Differentiate the risk with respect to $c$ and equating to zero, the minimizing $c$ must satisfies the following equation

$$
E_{\tau}\left\{\frac{\tau^{n}}{(n \tau-a)\left(\tau-a c_{0}\right)^{n}}\right\}=r,
$$

Yielding the best affine equivariant estimator $\delta_{\text {equivariant }}=\delta_{0}=\phi_{0}(Z) S$, where

$$
\phi_{0}(Z)=Z+c_{0} .
$$

\section{Improved Estimator}

For improving upon $\delta_{0}$, we study scale equivariant estimator $\delta=\phi(Z) S$. The risk of $\delta$ depends on $(\mu, \sigma)$ through $\frac{\mu}{\sigma}$, so without loss of generality one can take $\sigma=1$ and write

$$
R(\delta, \mu)=E_{\mu}\left\{E_{\mu}[L(\phi(Z) S, \theta) \mid Z=z]\right\} .
$$

The minimization of $R(\delta, \mu)$ leads to the following equation

$$
E_{\mu}\left[S \mathrm{e}^{a c S} \mid Z=z\right]=\mathrm{e}^{-a(\mu+k)} E_{\mu}[S \mid Z=Z] .
$$

let $z>0$, then the conditional density of $S$ given $Z=z>0$ is proportional to

$$
\frac{S^{n-1}}{(1+S(1+n z)-n \mu)^{n+r}} ; S>\max \left\{0, \frac{\mu}{z}\right\} \text {. }
$$

Consider now $\mu \leq 0$ and fix $z>0$, then setting 


$$
q(S ; \mu)=\frac{S^{n}}{(1+S(1+n z)-n \mu)^{n+r}} .
$$

From (12) we compute the following expectations as follows

$$
E_{\mu}(S \mid Z=z)=\int_{0}^{\infty} q(s ; \mu) \mathrm{d} s=\frac{1}{(1+n z)^{n+1}(1-n \mu)^{r-1}} \int_{0}^{1} u^{n}(1-u)^{r-2} \mathrm{~d} u
$$

and

$$
\begin{aligned}
& E_{\mu}\left(S \mathrm{e}^{a c S} \mid Z=z\right)=\int_{0}^{\infty} \mathrm{e}^{a c S} q(s ; \mu) \mathrm{d} s=\frac{1}{(1+n z)^{n+1}(1-n \mu)^{r-1}} \\
& \int_{0}^{1} \mathrm{e}^{a c \frac{1-n \mu}{1+n z} \cdot \frac{u}{1-u}} u^{n}(1-u)^{r-2} \mathrm{~d} u,
\end{aligned}
$$

where $u=\frac{S(1+n z)}{1+S(1+n z)-n \mu}$. Hence (12) becomes

$$
\int_{0}^{1} \mathrm{e}^{a c \frac{1-n \mu}{1+n z} \cdot \frac{u}{1-u}} u^{n}(1-u)^{r-2} \mathrm{~d} u=\mathrm{e}^{-a(\mu+k)} \frac{\Gamma(r-1) n !}{\Gamma(n+r)}
$$

any $c=\phi(Z)$ satisfying (15) minimizes $R(\delta, \mu)=E[E(L(\delta, \theta) \mid Z)]$, for $\mu \leq 0$ and $Z>0$. Now, let $\mu>0$ and fix again $Z>0$, then $S>\frac{\mu}{Z}, q(S, \mu)=\frac{S^{n}}{[1+S(1+n Z)-n \mu]^{n+r}}$.

So we have

$$
\begin{aligned}
& E_{\mu}[S \mid Z=z]=\int_{\mu / z}^{\infty} q(S ; \mu) \mathrm{d} s=\frac{1}{(1+n z)^{n+1}(1-n \mu)^{r-1}} \\
& \int_{\frac{z+\mu(1+n z)-n \mu z}{1} u^{n}(1-u)^{r-2} \mathrm{~d} u}
\end{aligned}
$$

and

$$
\begin{aligned}
& E_{\mu}\left[S \mathrm{e}^{a c S} \mid Z=z\right]=\int_{\mu / z}^{\infty} \mathrm{e}^{a c S} q(S ; \mu) \mathrm{d} s=\frac{1}{(1+n z)^{n+1}(1-n \mu)^{r-1}} \\
& \int_{\frac{\mu(1+n z)}{z+\mu(1+n z)-n \mu z}}^{1} \mathrm{e}^{a c S} u^{n}(1-u)^{r-2} \mathrm{~d} u
\end{aligned}
$$

and hence (7) becomes

$$
\int_{\frac{\mu(1+n z)}{z+\mu(1+n z)-n \mu z}}^{1} u^{n}(1-u)^{r-2} \mathrm{~d} u=\int_{\frac{\mu(1+n z)}{z+\mu(1+n z)-n \mu z}}^{1} \mathrm{e}^{a c S} u^{n}(1-u)^{r-2} \mathrm{~d} u
$$

any $c=c(\mu)$ satisfying (16) minimizes $R(\delta, \mu)=E[E[L(\delta, \theta) \mid Z]]$ for $\mu>0$ and $Z>0$ [14]. Now for deriving an improved equivariant estimator upon this we must find a bound for $c$ in formula (15) and (16). As we can not derive c from Equations (15) and (16) explicitely, this would not be achieved.

\section{Acknowledgements}

The grant of Alzahra University is appreciated.

\section{References}

[1] Lomax, K. (1954) Business Failures: Another Example of the Analysis of Failure Data. Journal of the American Statis- 
tical Association, 94, 847-852. http://dx.doi.org/10.1080/01621459.1954.10501239

[2] Bryson, M. (1974) Heavy-Tailed Distributions: Properties and Tests. Technometrics, 16, 61-68. http://dx.doi.org/10.1080/00401706.1974.10489150

[3] Arnold, B. (1983) Pareto Distribution. International Cooperative Publishing House, Silver Spring Maryland.

[4] Johnson, N., Kotz, S. and Balakrishnan, N. (1994) Continous Univariate Distributions. Vol. 1, 2nd Edition, Wiley \& Sons, New York.

[5] Lindley, D. and Singpurwalla, N (1986) Multivariate Distributions for the Life Lengths of Components of a System Sharing a Common Environment. Journal of Applied Probability, 23, 418-431. http://dx.doi.org/10.2307/3214184

[6] Nayak, Tk. (1987) Multivariate Lomax Distribution: Properties and Usefulness in Reliability Theory. Journal of Applied Probability, 24, 170-177. http://dx.doi.org/10.2307/3214068

[7] Kotz, S., Balakrishnan, N. and Johnson, N.L. (2000) Continuous Multivariate Distributions, Vol. 1, Models and Applications, 2nd Edition, Wiley \& Sons, New York.

[8] Petropoulos, C. and Kourouklis, S. (2004) Improved Estimation of Extreme Quantiles in the Multivariate Lomax (Pareto II) Distribution. Metrika, 60, 15-24. http://dx.doi.org/10.1007/s001840300293

[9] Petropoulos, C. and Kourouklis, S. (2001) Estimation of an Exponential Quantile under a General Loss and an Alternative Estimator under Quadratic Loss. Annals of the Institute of Statistical Mathematics, 53, 746-759. http://dx.doi.org/10.1023/A:1014648819462

[10] Rukhin A. and Strawderman, W. (1982) Estimating a Quantile of an Exponential Distribution. Journal of the American Statistical Association, 77, 159-162. http://dx.doi.org/10.1080/01621459.1982.10477780

[11] Varian, H.R. (1975) A Bayesian Approach to Real Estat Assessment. In: Fienberg, S.E. and Zellner, A., Eds., Studies in Bayesian Econometric and Statistics: In Honor of Leonard J. Savage, North Holland, Amesterdam, 195-208.

[12] Zellner, A. (1986) Bayesian Estimation and Prediction Using Asymmetric Loss Function. Journal of American Statistical Association, 81, 446-451. http://dx.doi.org/10.1080/01621459.1986.10478289

[13] Stein, C. (1964) Inadmissibility of the Usual Estimator for the Variance of a Normal Distribution with Unknown Mean. Annals of the Institute of Statistical Mathematics, 16, 155-160. http://dx.doi.org/10.1007/BF02868569

[14] Brewster, J.F. and Zidek, J.V. (1974) Improving on Equivariant Estimators. Annals of Statistics, 2, 21-38. http://dx.doi.org/10.1214/aos/1176342610 